\title{
Rectal Cancer: The Role of Radiotherapy
}

\author{
Paul G. Lidder Ken B. Hosie \\ Surgical Directorate, Derriford Hospital, Plymouth, UK
}

\section{Key Words}

Carcinoma, rectal $\cdot$ Radiotherapy $\cdot$ Mesorectal excision

\begin{abstract}
Surgery is the definitive tool in the management of patients with rectal carcinoma. Early failure of treatment remains problematic and is manifest in local recurrence. Development of surgical techniques and inclusion of other treatment modalities aim to reduce the incidence of local recurrence and improve survival. This article examines the role of radiotherapy in the management of patients with rectal carcinoma and explores the controversies that exist in its application. The evidence for the use and benefits of radiotherapy in adjuvant and neoadjuvant settings is reviewed, and its application in association with total mesorectal excision considered.
\end{abstract}

Copyright @ 2005 S. Karger AG, Basel

\section{Introduction}

There are 14,000 new cases of rectal cancer diagnosed in the UK per annum [1]. In 2001, rectal cancer accounted for over 5,000 UK deaths [1].

Surgery remains the mainstay of treatment. Despite a 'curative' resection, 5-year survival remains only 50-60\% [2-4]. It is consistently reported that one-third of all treatment failures are secondary to local recurrence [3-6]. Furthermore, only one-quarter of patients with recurrent disease can hope to be cured, and the 5-year mortality exceeds $80 \%$ [6, 7]. Such disappointing results have encouraged the application of adjuvant treatments, such as radiotherapy (RT).

The first successful treatment of rectal cancer employing irradiation was reported by Symonds in 1914 [8]. Advancements in this field have led to the development of several techniques of administration including: external beam radiation, endocavitary radiation (contact X-ray and brachytherapy), and combinations of both.

Anatomical distinction between colonic and rectal tumours is important from an oncological perspective. The rectum is the portion of large bowel extending below the peritoneal reflection to the anus [9]. Approximately $13 \mathrm{~cm}$ in length, the upper margin lies at the level of the third sacral vertebra [10]; intraoperatively this is recognised by

Paul G. Lidder

Mr. Hosie's Office, Derriford Hospital

Surgical Directorate, Level 7

PL6 8DH Plymouth (UK)

Tel. +44 1752 763964, Fax +44 1752 763436, E-Mail pglidder@hotmail.com E-Mail karger@karger.ch www.karger.com 
Table 1. Summary of current adjuvant/neoadjuvant therapies for rectal carcinoma

\begin{tabular}{llll}
\hline Regimen & $\begin{array}{l}\text { Short course } \\
\text { preoperative RT }\end{array}$ & $\begin{array}{l}\text { Long course } \\
\text { preoperative RCT }\end{array}$ & $\begin{array}{l}\text { Long course } \\
\text { postoperative RCT }\end{array}$ \\
\hline $\begin{array}{l}\text { Timing } \\
\text { Fraction }\end{array}$ & $\begin{array}{l}1 \text { week preoperative } \\
5 \text { Gy } \times 5 \text { days }\end{array}$ & $\begin{array}{l}4-6 \text { weeks preoperative } \\
1.8 \mathrm{~Gy} \times 5.5 \text { weeks }\end{array}$ & postoperative \\
\hline
\end{tabular}

confluence of the taenia. Cancers above the reflection are managed by a colonic paradigm, whereas, rectal cancers frequently employ adjuvant therapies in their routine management [9]. Treatment failure of resected colon cancer usually arises from the clinical progression of previously undetected metastases [9]. In rectal cancer there is the additional substantial risk of local failure due to unresected local microscopic disease [9].

Adjuvant RT aims to improve survival and reduce local recurrence by treating any residual microscopic disease [9]. Achieving better local control presents not only a singular opportunity to improve long-term survival, it reduces a distressing, debilitating and painful mode of death [5].

\section{Role of Radiotherapy}

Key issues regarding the use of adjuvant RT in the management of rectal cancer are: (1) Which cancer patients benefit from RT? (2) Pre- or postoperative RT? (3) Short or long course RT? (4) The role of RT with total mesorectal excision (TME)-based surgery. (5) Side effects of RT.

\section{Current Radiotherapeutic Regimens}

There are three commonly applied therapeutic regimens [11]: (1) Short course preoperative radiotherapy: well-tolerated, practical and financially efficient it is the most popular method in Europe; (2) Long course preoperative radiotherapy \pm chemotherapy $45 \mathrm{~Gy}$ delivered in 1.8-Gy daily fractions over 5.5 weeks, followed by a 5.4Gy pelvic boost to the primary tumour, and (3) 'Long course' postoperative radiotherapy \pm chemotherapy. Predominant practice in USA, it is delivered concurrently with 5-FU-based chemotherapy. In Europe this regimen is applicable to patients who did not receive preoperative adjuvant RT deemed to be at high risk following their surgery (table 1).

\section{Indications for Radiotherapy}

At present there is a variable amount of evidence to support the use of adjuvant RT in three situations: firstly, as an adjunct to resective surgery to reduce local recurrence [12]; secondly, to facilitate resection of a tumour considered unresectable at presentation; 'downstaging', or to 'downsize' a resectable cancer to enable a sphincterpreserving operation [12], and thirdly, as a palliative therapy in those patients presenting with inoperable disease or in whom comorbidity precludes operation [12].

\section{Role of Radiotherapy in Reducing Local Recurrence}

Despite 'curative' surgery, numerous series have reported local recurrence rates from 15 to $50 \%$ following conventional surgery. The majority of this data is extracted from randomised control trials (RCTs) which included conventional non-standardised surgery alone arms vs. adjuvant therapy $[13,14]$. Conventional surgery describes non-standardised blunt dissection of the pelvic fascia. In 1986 it was recognised that local recurrence was in the main a consequence of incomplete excision of radial spread [15]. Quirke et al. [15] demonstrated that $85 \%$ of local recurrences developed in patients with microscopically involved circumferential margins. Conversely, the recurrence rate with a negative lateral margin was only $3 \%$ [15]. This knowledge instigated a revolution in the practice of colorectal resection: Pioneered by Heald this has led to the current gold standard - TME (total mesorectal excision). Several RCTs have confirmed that TME-based surgery has significantly improved the survival of patients with colorectal cancer and reduced local recurrence rates to single figures [14]. The bulk of data regarding the benefits of adjuvant $\mathrm{RT}$ in reducing local recurrence is elicited from trials in which oncologically inferior conventional surgery was performed. This in part explains the existence of some of the controversies that surround perioperative RT today. 
Which Patients Would Potentially Benefit from

Radiotherapy?

A meta-analysis of 14 RCTs demonstrates that preoperative RT significantly improves survival and 5-year cancer-specific survival with impressive reductions in local recurrence rate [2]. Complications in the immediate postoperative period were significantly increased, particularly with the wound and sepsis although there was no increase in anastomotic dehiscence [2]. Subgroup analysis failed to show survival benefit in early (Dukes A) cancer and benefit was most pronounced in Dukes B and C disease [2]. Preoperative staging utilising computed tomography, magnetic resonance imaging and transrectal ultrasound will better predict those patients with mesorectal or nodal disease, all of whom are at higher risk of recurrent disease $[16,17]$. It is this group of patients in whom adjuvant therapy should be targeted.

\section{Should Radiotherapy Be Given before or after}

Surgery?

Timing of adjuvant RT presents a therapeutic conundrum. A key advantage of postoperative RT is the optimal selection of patients based on intraoperative findings and histopathological evidence [3]. Analysis of 6 RCTs examining postoperative RT failed to demonstrate survival benefit [3]. Only 1 showed a reduction in local recurrence and almost a third reported non-compliance [3]. Both acute and late toxicity were significant, the small bowel being affected in $40 \%$ of patients [3]. Aside from issues of compliance, and higher toxicity, postoperative RT may be less effective due to disturbed vascularisation of the pelvis reducing cellular oxygen tension and sensitivity to $\mathrm{RT}$. Irradiation of the neorectum also leads to a poorer functional outcome [17].

Combined postoperative chemoirradiation schemes used in the USA have shown significant benefit over surgery alone, postoperative RT alone or postoperative chemotherapy [3]. Both local and distal recurrence is reduced and survival enhanced [3]. Based on three of these trials [18-20] the National Institutes of Health in 1990 endorsed postoperative radiochemotherapy as the standard management of patients with stage II and III disease in the USA [21]. This remains the situation today (table 2).

In Europe, preoperative RT has gained acceptance as the standard adjuvant therapy for T3 disease [21]. Potential advantages include decreased tumour seeding at surgery, less acute toxicity, increased compliance, and improved therapeutic index due to increased radiosensitivity. Additionally, the pelvic field contains minimal small bowel and the anastomosis will be fashioned with proxi- mal bowel that has not been irradiated [22]. The primary disadvantage is overtreatment of patients with early (T12 N0) disease and the potential loss of important staging evidence. Additionally, patients with metastatic spread will be subjected to inappropriate irradiation if not diagnosed preoperatively [9]. Seven of 8 RCTs recruiting almost 5,000 patients demonstrated a significant decrease in local recurrence rates and 3 trials showed survival gain. This was most notable in the large Swedish Rectal Cancer Trial [28]. All patients with T1-3 disease were randomised to either short course RT over 1 week followed by surgery a week later, or surgery alone. Local recurrence in the RT group was 12 vs. $27 \%(\mathrm{p}<0.001)$ [28]. Correspondingly, 5 -year survival was 58 vs. $48 \%(\mathrm{p}=0.04)$ for those receiving neoadjuvant RT [28].

\section{Is Radiotherapy Required if Surgery Is Optimised?}

Historical data based on conventional non-standardised colorectal surgery reports high rates of local recurrence. Surgery has evolved and with TME-based dissection recurrence rates are frequently single figures comparable with the recurrence rates reported following adjuvant $\mathrm{RT}$.

It has been demonstrated that the surgeon is an independent variable for outcome and that local recurrence may be regarded as a failure of technique [23, 24]. In specialist centres experienced in TME, rates of local recurrence as low as $2.6 \%$ are quoted. Generally, rates $<10 \%$ are acceptable for TME-based surgery. With this marked reduction in local recurrence, many have postulated that RT is unnecessary if surgery is optimised, particularly, since RT carries a morbidity and mortality risk $[25,26]$.

The Dutch Colorectal Cancer Group (DCRCG) showed a significant reduction in local recurrence from 16 to $9 \%$ with the introduction of TME [14], and initial analysis of accrued survival data confirms similar benefit. This is comparable with the results of several other series reporting local recurrence rates of $4-11 \%$ [27]. Between 1996 and 1999, the DCRCG recruited 1,861 patients for a multicentre prospective RCT (CKVO-95-04) examining the role of short course preoperative RT in conjunction with TME surgery. Benefit was again shown. The local recurrence rate for surgery with adjuvant RT was 2.4 vs. $8.2 \%$ for surgery alone $(p<0.001)$ [33]. The magnitude of benefit was greatest in node-positive patients. In stage III disease, local recurrence fell from 15.0 to $4.3 \%$. Local recurrence dropped from 5.7 to $1.0 \%$ for stage II rectal cancers. Therefore, despite improvements in surgical practice, an additive benefit of RT exists although overall benefit is reduced $[28,33]$. In real terms this translates to 
Table 2. Summary of randomised trials investigated pre-/postoperative radio(chemo)therapies in rectal carcinoma

\begin{tabular}{|c|c|c|c|}
\hline Trial & $\begin{array}{l}\text { Radiotherapy total } \\
\text { dose, Gy/fractions }\end{array}$ & $\begin{array}{l}\text { Decreased local } \\
\text { recurrence }\end{array}$ & $\begin{array}{l}\text { Increased } \\
\text { survival }\end{array}$ \\
\hline \multicolumn{4}{|l|}{ Preoperative } \\
\hline Norway [22] & $31.5 / 18$ & No & No \\
\hline Stockholm [23] & $25 / 5$ & Yes $(p<0.01)$ & No \\
\hline EORTC [24] & $34.5 / 15$ & Yes $(p=0.03)$ & No \\
\hline Manchester [25] & $20 / 4$ & Yes $(\mathrm{p}<0.01)$ & Yes $(p=0.03)$ \\
\hline Stockholm II [26] & $25 / 5$ & Yes $(\mathrm{p}<0.01)$ & $\operatorname{Yes}(p=0.01)$ \\
\hline St Marks RCG [27] & $15 / 3$ & Yes $(p=0.05)$ & No \\
\hline SRCT [28] & $25 / 5$ & Yes $(p=0.001)$ & Yes $(p=0.004)$ \\
\hline MRC I [29] & $20 / 10$ & No & No \\
\hline MRC II [30] & $40 / 20$ & Yes $(p=0.04)$ & No \\
\hline VASOG I [31] & $20 / 10$ & No & No \\
\hline VASOG II [23] & $31.5 / 18$ & - & No \\
\hline Dutch TME [33] & $25 / 5$ & Yes $(\mathrm{p}<0.001)$ & Yes \\
\hline \multicolumn{4}{|l|}{ Postoperative } \\
\hline GITSG [18] & $40-48 / 20-28$ & No & No \\
\hline ECOG [34] & $45 / 25$ & & No \\
\hline NSABP R-01 [20] & $46-47 / 26-27$ & No & No \\
\hline ANZ [35] & $45 / 25$ & No & No \\
\hline Rotterdam [36] & $50 / 25$ & No & No \\
\hline Odense [37] & $50 / 25$ & No & No \\
\hline MRC 3 [38] & $40 / 20$ & Yes $(p=0.001)$ & No \\
\hline \multicolumn{4}{|l|}{ Postoperative RCT } \\
\hline GITSG 7175 [39] & $+5-\mathrm{FU} \pm$ semustine & No & Yes $(p=0.005)$ \\
\hline NCCTG $[40]$ & $+5-\mathrm{FU} \pm$ semustine & Yes $(p=0.03)$ & Yes $(p=0.02)$ \\
\hline Intergroup US Trial [41] & $+5-\mathrm{FU}$ & No & Yes $(p=0.005)$ \\
\hline Tveit et al. [42] & $+5-\mathrm{FU}$ & Yes $(p=0.01)$ & Yes $(\mathrm{p}<0.05)$ \\
\hline Cafiero et al. [43] & +5 -FU/levamisole & No & No \\
\hline NSABP-R-01 [44] & $\begin{array}{l}\text { + Methyl-CCNU/ } \\
\text { vincristine/5-FU }\end{array}$ & No & Yes $(p=0.05)$ \\
\hline NSABP-R-02 & $+5-\mathrm{FU}$ & Yes $(p=0.02)$ & No \\
\hline
\end{tabular}

5-FU = 5-Fluorouracil.
6 patients benefiting for every 100 irradiated. No survival benefit was demonstrated at the 2-year follow-up [33].

\section{Role of Radiotherapy in Enhancing Operability and Sphincter Preservation \\ 'Downstaging'}

Up to $15 \%$ of patients diagnosed with rectal cancer harbour a tumour that is invading adjacent tissues such as the base of the bladder or bony pelvis, which are not readily resectable [29]. Attempts at surgery would leave a residual burden of disease. RT is used to downstage a primarily unresectable tumour to facilitate resection. Obviously, only neoadjuvant RT can produce this response.

Downstaging is dependent on fraction size, total dosage and correlates to the overall treatment time, i.e. the interval between commencing irradiation and subsequent resection [30]. There are no RCTs to demonstrate the efficacy of downstaging although little controversy exists for this use. Conventionally, $50 \mathrm{~Gy}$ in 2-Gy fractions are administered over a 5-week period. Chemotherapy is often added to RT, but again there is no supportive evidence that combination therapy is superior although toxicity is increased [4].

\section{'Downsizing'}

The primary objective when managing patients with colorectal cancer is to achieve cure. From the patient's perspective this is allied with quality of life thereafter. Often this is synonymous with sphincter conservation and avoidance of a permanent stoma [31]. 
There were two major advances that preluded the era of sphincter-conserving surgery. Firstly, greater understanding of anorectal physiology showed that the distal 1$2 \mathrm{~cm}$ of rectum and even the upper internal sphincter are not absolute requisites for continence [32]. Empowered by better stapling devices, lower anastomoses became feasible. Secondly, it was recognised that local recurrence was a consequence of incomplete excision of lateral mesorectal involvement. Soon the 'requisite' $5-\mathrm{cm}$ distal resection margin was being questioned. In 1990, Karanjia et al. [33] reported 'a close shave' at anterior resection suggesting a $1-\mathrm{cm}$ distal resection margin to be oncologically acceptable. Intraparietal distal spread is usually limited to 1-2 $\mathrm{cm}$, and a $2-\mathrm{cm}$ margin is safely accepted by many surgeons [34]. Proponents of RT have frequently advocated preoperative RT as a tool for downstaging disease securing a safety margin facilitating sphincter preservation. This is an inaccurate description of the effect of RT in this instance, which is to 'shrink' the tumour and devitalise tumour cells that may be left behind. This does not affect the stage and is more appropriately termed 'downsizing' [21].

Although this concept is widely held and preoperative RT recommended to facilitate sphincter preservation, trial evidence is weak. Most data to support these claims is derived from phase II trials and conclusions should be interpreted with due caution. Wagman et al. [35] reported a series of 36 patients prospectively deemed to require abdominoperineal resection following surgical assessment. The RT regimen consisted of 4,680 cGy at 180 cGy/day to the whole pelvis with a $360-\mathrm{cGy}$ boost to the tumour. Surgery was performed 4-5 weeks later. Seventyseven percent underwent low anterior resection \pm coloanal anastomosis. Five-year actuarial disease-free survival was $60 \%$, and $85 \%$ reported 'good' or 'excellent' sphincter function. Rouanet et al. [36] reported similar findings.

Considering that the sphincter-preserving benefits of preoperative RT are so widely popularised it is perhaps surprising that supportive evidence is so scanty. A possible explanation is that within Europe the bulk of preoperative RT regimens follow the Swedish model. Namely, intensive short course therapy (5 Gy $\times 5$ days) followed by surgery 1 week later. This may provide insufficient time for shrinkage. The Lyons R90-01 RCT randomised 201 patients into a short interval group or a long interval group. There was a non-significant trend towards sphincter preservation if surgery was delayed 6-8 weeks (long interval) following RT $(13 \times 3 \mathrm{~Gy})$ as opposed to resection performed within 2 weeks of completion (short inter- val) [37]. The authors concluded that delaying surgical resection until the fifth or sixth week increased downsizing facilitating sphincter preservation. No detrimental effects on morbidity or mortality rates nor local recurrence or 5-year survival resulted from the delay in surgery. However, it is recognised that pelvic irradiation has a negative effect on functional outcome [38]. In the Swedish RCT, $30 \%$ of preoperatively irradiated patients had some degree of bowel dysfunction compared with $10 \%$ in the surgery alone group [28]. The most frequent complaints are of incontinence to loose stool and urgency. These detrimental effects are dose dependent, but the exact contribution of RT to these symptoms is complex.

Certainly 'anterior resection syndrome', characterised by frequency, urgency and decreased control, becomes more pronounced the lower the anastomosis. The internal sphincter comprised of smooth muscle innervated by the myenteric plexus appears to be more susceptible to the dose-dependent deleterious effects of RT, than the striated muscle of the external sphincter served by the pudenal nerve. Patients undergoing coloanal anastomosis and J-pouch reconstruction seem similarly at risk from anorectal dysfunction following irradiation. These effects are most pronounced with postoperative RT schedules. Birnbaum et al. [39, 40], examined the short- and longterm impact of preoperative RT and reported minimal effects on sphincter function.

Preservation of the sphincter mechanism is deemed a means of improving quality of life in colorectal cancer, however, it should be remembered that a well-functioning colostomy will achieve this better than a poorly-functioning sphincter.

\section{Role of Radiotherapy in Palliation}

There are several groups in whom RT may form the sole treatment. It may be inappropriate for frail medically unfit patients to undergo surgery, or surgery may not be an option if disease is advanced.

Although not widely available, contact X-ray is a favourable method of control for T1 lesions in frail elderly patients [8]. It is a fully ambulatory treatment performed in the knee-chest lateral position. This inexpensive daycase procedure administered in 4-5 fractions is suited to those with a low risk of lymph node metastases [8]. Lesions should be well or moderately differentiated, measure $<4 \mathrm{~cm}$ and be polypoidal without ulceration. Transrectal ultrasound may provide additional staging information and in appropriately targeted patients local control rates vary between 80 and $90 \%$ [8]. 
Table 3. Chronological development of radiation-induced side effects

\begin{tabular}{lll}
\hline Early 0-3 months & Late 6-24 months & Delayed 2-40 years \\
\hline Diarrhoea & Bleeding & Malabsorption \\
Tenesmus & Fistulae & Fistulae \\
Bleeding & Obstruction & Second primary \\
Obstruction & & Tumour \\
Perforation & & \\
\hline
\end{tabular}

As a palliative treatment, external beam RT is particularly effective in reducing pain and bleeding [7, 12, 41]. These symptoms are particularly alarming and distressing and the benefit of alleviation cannot be overstated. It is however less effective in managing altered bowel habit [13], discharge and neurological symptoms.

As a palliative measure, dosage and fractionation schedules are less certain. Single fractions of 8-10 Gy may be suitable for frail patients [2]. In fitter patients, higher doses of the order of 20-30 Gy in 5-10 fractions are often used, but evidence for such regimens is scarce [2].

Survival for recurrent disease treated principally by external beam RT is poor; approaching 5\% for 5 years. Similarly, alleviation of symptoms is short-lived with median time to disease progression approximately 5 months and median survival 14 months.

Figures are slightly better for those who receive external beam RT for local recurrence treated principally with surgical resection. Five-year survival and local recurrence rates are reported as 20 and $15 \%$ respectively [11]. These rates are doubled if regimens using $50 \mathrm{~Gy}$ or higher are used. Endocavitary RT may be similarly be used in the palliative setting. Endocavitary radiation therapy describes two distinct modalities. Irradiation may administered by an intraluminal emitter inserted into the rectum [8]. Alternatively, iridium implants are placed within the tumour bulk by endoluminal or transperineal approaches [8]. In the palliative setting, endocavitary RT is a safe, easy and efficient method of treating bleeding [8]. Reports of local control vary from 35 to $45 \%$ [8].

As a palliative treatment, the benefits of RT must be weighed against its side effects. For advanced symptomatic pelvic disease it can alleviate distressing symptoms although benefit may be short-lived. In such cases complete remission is rarely achieved. For mobile tumours in patients unfit for surgery it is more effective and presents an option for cure in $25 \%$.
In inoperable carcinomas alternative palliative treatments exist in addition to RT; these include thermal, laser and photodynamic ablative modalities, and in proximal rectal tumours stents may be utilised. For those patients who are fit, surgical resection is preferred.

\section{Morbidity and Mortality Associated with Radiotherapy}

Adverse effects of RT occur to some degree in most patients but are usually self-limiting. Several factors influence the frequency and severity of symptoms. These include short or long course regimens, pre- or postoperative administration, field, port and fraction sizes and the administration of concomitant chemotherapy (table 3 ).

Short-term complications are usually transient and include lethargy, nausea, diarrhoea (7-30\%), skin erythema and desquamation and cystitis. With high dose $(5 \times$ $5 \mathrm{~Gy}$ ) preoperative short course regimens, rates of perineal wound infections following abdominoperineal resection are doubled [16]. The Swedish Rectal Cancer Group, EORTC and UK MRC trials did not demonstrate any higher incidence of wound infection or anastomotic complications (dehiscence, stenosis) following preoperative RT.

Six percent of patients receiving short course preoperative RT in the Uppsala trial developed acute lumbosacral plexopathy [42]. This is usually short-lived but may persist for several months in the minority.

Long-term effects include thromboembolic, femoral neck and pelvic fractures, delayed wound healing and fistulae, and intestinal obstruction [16]. Such events were reported in the Stockholm I trial which employed a large field 2-port technique to administer short course preoperative RT [43]. The Stockholm II trial followed a 4-port technique and did not report an increase in such complications. Widespread adoption of a four-port technique and a reduced field volume minimises the deleterious effects of RT by minimising irradiation of healthy uninvolved tissues [44].

Considering postoperative RT, complications are more common: diarrhoea (8-48\%), nausea (4-17\%), skin reactions $(8-28 \%)$, cystitis $(6-12 \%)$, and fatigue $(14 \%)$. Up to $50 \%$ of patients fail to complete their adjuvant therapy because of these adverse effects. Whilst generally mild, a subgroup of patients experience severe symptoms requiring hospitalisation, particularly with increasing age.

Late complications more frequently include small bowel obstruction (5-11\%) and ileus and perforation are 
associated with high dosage regimens. Small bowel complications may be related to postoperative adhesions and prolapsing of dependent small bowel within the pelvis. The use of multiple fields to minimise the volume of small bowel irradiated is associated with a significant reduction in these complications, although not completely avoidable (table 4).

Further drawbacks of postoperative regimens include delay in administration because of incomplete wound healing and 'postoperative fatigue'. The oncological effects of delay are little known although concerns of tumour cell repopulation and relapse exist. Concurrent administration of chemotherapy with postoperative RT further increase both early and late morbidity.

Mortality rates for patients receiving neo/adjuvant RT or combined chemoradiotherapy (CRT) vary from 4 to $18 \%$. Rates are higher with combined modality treatment and associated with increasing age and comorbidity.

\section{Conclusion}

RT has a justifiable position in the treatment of rectal cancer. It is a beneficial adjunct in the management of resectable disease and these benefits are additive to the benefits already observed in TME-based surgery. The relative benefits are, however, lessened by optimised surgery and therefore case selection rather than blanket administration is appropriate. Cross-sectional imaging as well as transrectal ultrasound are therefore key tools in deciding which patients are at highest risk of local recurrence and will experience maximal benefit of RT. Judicial use will lessen the impact of the detrimental toxic effects pelvic irradiation can produce. In the future, molecular markers such as K-ras and p53 may be similarly crucial in identifying high-risk candidates [9].

Currently, there is a transatlantic dichotomy in the implementation of RT. In the USA, postoperative combination chemoirradiation is preferred. This reduces the risk of inappropriate administration but compliance is poorer, efficiency on a dose-correlated basis is lower and toxicity is higher. In Europe short course RT is preferred for selected cases; it is more efficient with lower toxicity and is better tolerated.

Whilst the role of RT as an adjuvant and palliative treatment in rectal cancer is well established, further evidence is required to establish the most efficacious administration. The National Institutes of Health recommend postoperative CRT as the treatment of choice in stage II and III resectable disease [7]. A similar stance is taken in
Table 4. Incidence of frequently reported complications (\%) where stated for preoperative radiotherapy, preoperative radiochemotherapy and postoperative radiochemotherapy regimens

\begin{tabular}{|c|c|c|c|}
\hline Morbidity/mortality & $\begin{array}{l}\text { Preoperative } \\
\text { RT, \% }\end{array}$ & $\begin{array}{l}\text { Preoperative } \\
\text { RCT, } \%\end{array}$ & $\begin{array}{l}\text { Postopera- } \\
\text { tive RCT, } \%\end{array}$ \\
\hline Death & $0-9$ & $4-9$ & $0-4$ \\
\hline Diarrhoea & $7-30$ & $33-39$ & $11-41$ \\
\hline Nausea & & $10-18$ & 4-38 \\
\hline Ileus & 5 & & $3-5$ \\
\hline Small bowel obstruction & $4-13$ & & $4-5$ \\
\hline Anastomotic stenosis & & & \\
\hline Anastomotic leak & $7-15$ & & \\
\hline Wound infection & $5-14$ & & \\
\hline Perineal wound infection & $8-26$ & & \\
\hline Pelvic sepsis & $8-17$ & & \\
\hline Radiation cystitis & 28 & & 11 \\
\hline Skin reaction & $2-5$ & & $3-33$ \\
\hline Thromboembolism & $7-13$ & & \\
\hline
\end{tabular}

Germany [7]. Elsewhere in Europe preoperative RT alone is favoured and advocated by the Paris Consensus Conference (1994) [7]. These conflicting approaches are based on data accrued over a decade ago. More recently, several randomised trials are aiming to establish best practice. Two US studies comparing pre- and postoperative CRT were commenced. Unfortunately, both NSABP R-03 and INT 0147 were closed prematurely due to poor accrual. A similar German trial CAO/ARO/AIO 94 followed a similar methodology, and recently completed recruitment [3, $7,9]$.

Twelve-month analysis of NSABP R-03 data revealed that the percentage of patients undergoing sphincter-saving surgery without evidence of disease was 44\% (preoperative CRT) compared with 33\% (postoperative CRT) [9]. Preliminary data from the German CAO/ARO/AIO 94 suggests preoperative chemoradiotherapy does not increase postoperative morbidity/mortality. This trial may go on to provide the best data yet on whether neoadjuvant therapies confer a sphincter-sparing advantage.

Finally, the ongoing British Medical Research Council (MRC) CR07 trial comparing short course preoperative RT and postoperative CRT may deliver the best data which will end the controversy over pre- vs. postoperative adjuvant therapy [45].

Rectal Cancer: Role of Radiotherapy

Dig Surg 2005;22:41-49 


\section{References}

1 Cancer Research UK: www.cancerresearchuk. org/statistics.

$\checkmark 2$ Camma C, Giunta M, Fiorica F, Pagliaro L, Craxi A, Cottone M: Preoperative radiotherapy for resectable rectal cancer: A meta-analysis. JAMA 2000;284:1008-1014.

$\checkmark 3$ Bosset JF, Mantion G, Lorchel F, Magnin V, Pelissier EP, Gerard JP, Horiot JC: Adjuvant and neoadjuvant radiation therapy for rectal cancer. Semin Oncol 2000;27(suppl 10):6065.

-4 Pahlman L: Perioperative radiotherapy in the management of rectal cancer. Can J Gastroenterol 2001;15:259-263.

5 Bujko K, Nowacki MP: Emerging standards of radiotherapy combined with radical rectal cancer surgery. Cancer Treat Rev 2002;28:101113.

-6 Kendal WS, Cripps C, Viertelhausen S, Stern $\mathrm{H}$ : Multimodality management of locally recurrent colorectal cancer. Surg Clin North Am 2002;82:1059-1073.

$\checkmark 7$ Sauer R: Adjuvant and neoadjuvant radiotherapy and concurrent radiochemotherapy for rectal cancer. Pathol Oncol Res 2002;8:7-17.

$>8$ Gerard JP, Romestaing P, Ardiet JM, Mornex F: Endocavitary radiation therapy. Semin Radiat Oncol 1998;8:13-23.

$\checkmark 9$ Saltz LB, Minsky B: Adjuvant therapy of cancers of the colon and rectum. Surg Clin North Am 2002;82:1035-1058.

10 Snell RS: Clinical Anatomy for Medical Students, ed 4. Boston, Little, Brown, 1992.

$\checkmark 11$ Wong CS, Cummings BJ, Keane TJ, O’Sullivan $\mathrm{B}$, Catton $\mathrm{CN}$ : Results of external beam irradiation for rectal carcinomas locally recurrent after local excision or electrocoagulation. Radiother Oncol 1991;22:145-148.

12 Hatfield P, Sebag-Montefiore D: The use of radiotherapy in rectal cancer. Scand J Surg 2003;92:65-73.

13 Edwards DP, Mortensen NJ: Is radiotherapy for rectal cancer indicated if surgery is optimized? Eur J Surg 2001;27:442-445.

$\checkmark 14$ Kapitejin E, Putter H, Van de Velde CJH, et al: Impact of the introduction and training of total mesorectal excision on recurrence and survival in rectal cancer in The Netherlands. Br J Surg 2002;89:1142-1149.

15 Quirke P, Dixon MF, Durdley P, et al: Loca recurrence of rectal cancer due to inadequate surgical resection. Histopathological study of lateral tumour spread and surgical excision. Lancet 1986;ii:996-998.

16 Sebag-Montefiore D: Adjuvant radiation therapy for rectal cancer: Selecting the right cases. Curr Probl Cancer 2003;27:54-59.
17 Sobat H, Juretic A, Samija M: Combined modality therapy of rectal cancers. Ann Oncol 1999;10:99-103.

18 Gastrointestinal Tumour Study Group: Prolongation of the disease-free interval in surgically treated rectal carcinoma. N Engl J Med 1985;312:1464-1472.

19 Krook JE, Moertel CG, Gunderson LL, et al: Effective surgical adjuvant therapy for high risk rectal cancer. N Engl J Med 1991;324:709_ 715.

20 Fisher B, Wolmark N, Rockette H, et al: Postoperative adjuvant chemotherapy or radiation therapy for rectal cancer: Results from NSABP Protocol R-01. J Natl Cancer Inst 1988;80:2129.

21 Glimelius B: The role of radiotherapy in rectal cancer. Eur J Cancer 2001;37(suppl 7):203212.

22 Minsky BD: Preoperative radiation therapy followed by low anterior resection with coloanal anastomosis. Semin Radiat Oncol 1998;8: 30-35.

23 Seow-Choen F: Adjuvant therapy for rectal cancer cannot be based on the results of other surgeon. Br J Surg 2002;89:946-947.

24 Martling A, Cedermark B, Johansson H, Rutqvist LE, Holm T: The surgeon as a prognostic factor after the introduction of total mesorectal excision in the treatment of rectal cancer. $\mathrm{Br} \mathbf{J}$ Surg 2002;89:1008-1013.

25 Holm T, Rutqvist LE, Johansson H, Cedermark B: Postoperative mortality in rectal cancer treated with or without preoperative radiotherapy: Causes and risk factors. Br J Surg 1996;83:964-968.

26 Obi BS, Tjandra JJ, Green MD: Morbidities of adjuvant chemotherapy and radiotherapy for resectable rectal cancer: An overview. Dis Colon Rectum 1999;42:403-418.

27 Buwlow S, Christensen IJ, Harling H, et al: Recurrence and survival after mesorectal excision for rectal cancer. Br J Surg 2003;90:974980.

28 Colquhoun P, Wexner SD, Cohen A: Adjuvant therapy is valuable in the treatment of rectal cancer despite total mesorectal excision. J Surg Oncol 2003;83:133-139.

29 Glimelius B: The role of preoperative and postoperative radiotherapy in rectal cancer. Clin Colorectal Cancer 2002;2:82-92.

30 Marijnen CAM, Glimelius B: The role of radiotherapy in rectal cancer. Eur J Cancer 2002;38: 943-952.
31 Ahmad NR, Nagle DA: Preoperative radiation therapy followed by local excision. Semin Radiat Oncol 1998;8:36-38.

32 Tytherleigh MG, Mortensen NJ: Options for sphincter preservation in surgery for low rectal cancer. Br J Surg 2003;90:922-933.

33 Karanjia ND, Schache DJ, North WR, Heald $\mathrm{RJ}$ : 'Close shave' in anterior resection. $\mathrm{Br} \mathrm{J}$ Surg 1990;77:510-512.

34 Marijnen CAM, Glimelius B: The role of radiotherapy in rectal cancer. Eur J Cancer 2002;38: 943-952.

35 Wagman R, Minsky BD, Cohen AM, et al: Sphincter preservation with preoperative radiotherapy and coloanal anastomosis: Longterm follow-up. Int J Radiat Oncol Biol Phys 1997;39:167.

36 Rouanet P, Fabre JM, Dubois JB, et al: Conservative surgery for low rectal cancer after high dose radiation: Functional and oncologic results. Ann Surg 1995;221:67-73.

37 Glehen O, Chapet O, Adham M, et al: Longterm results of the Lyons R90-01 randomised trial of preoperative radiotherapy with delayed surgery and its effect on sphincter-saving surgery in rectal cancer. Br J Surg 2003;90:996998.

38 Gervaz PA, Wexner SD, Pemberton JH: Pelvic radiation and anorectal function: Introducing the concept of sphincter-preserving radiation therapy. J Am Coll Surg 2002;195:387-393.

39 Birnbaum EH, Dreznik Z, Myerson RJ, et al: Early effect of external beam radiation on anal sphincter: A study using anal manometry and transrectal ultrasound. Dis Colon Rectum 1992;35:757-761.

40 Birnbaum EH, Myerson RJ, Fry RD, Kodner IJ, Fleshman JW: Chronic effects of pelvic radiation therapy on anorectal function. Dis Colon Rectum 1994;37:909-915.

41 Wong CS, Brierley JD: External beam radiation therapy alone. Semin Radiat Oncol 1998; 8:3-12.

42 Frykholm GJ, et al: Acute lumbosacral plexopathy during and after preoperative radiotherapy of rectal adenocarcinoma. Radiother Oncol 1991;20:10-15.

43 Stockholm Rectal Cancer Study Group: Preoperative short-term radiation therapy in operable rectal carcinoma: A prospective randomised trial. Cancer 1990;66:49-55.

44 Bechtel J, Tepper J: Adjuvant radiation therapy of patients with rectal cancer. Clin Colorectal Cancer 2003;2:213-222.

45 Steel RJC, Sebag-Montefiore D: Adjuvant radiotherapy for rectal cancer. Br J Surg 1999;86: 1233-1234. 


\section{Invited Commentary}

Alexis Ulrich

Surgical Department, University of Heidelberg, Heidelberg, Germany

The authors present a thorough review about the role of radiotherapy in rectal cancer. Especially the introduction of the total mesorectal excision (TME) has improved the outcome of rectal cancer patients significantly by reducing the local recurrence rate. Interestingly, the outcome had been comparable to the standard operation combined with adjuvant (postoperative) radiochemotherapy, questioning the need of multimodal therapy concepts with the new technique. However, large randomised controlled trials indicate a benefit of multimodal concepts in patients with stage II or III disease. The trial of the Dutch Colorectal Cancer Group showed a significant reduction of the local recurrence rate by short-term preoperative radiotherapy compared to TME alone.

Another point of controversy, clearly demonstrated by the authors, is the time of application of radiotherapy. While in the USA adjuvant radiotherapy is preferred, Europeans favour the neoadjuvant concepts. Based on the available data, preoperative radiotherapy seems to be more effective in respect of tumour control as well as early and late toxicity. In the ARO trial comparing preoperative with postoperative radiochemotherapy, the local recurrence ( 7 vs. $11 \%$ ) and toxicity rates (acute 28 vs. $39 \%$; late 10 vs. $23 \%$ ) were significantly lower in the preoperative group. Of course, $18 \%$ of the patients undergoing preoperative RCT had probably been overstaged before the operation and unnecessarily received radiotherapy. On the other hand, up to $50 \%$ of the patients scheduled for postoperative radiotherapy do not complete or even start the treatment due to complications or delayed wound healing. In this context it seems to be adequate to favour the preoperative approach.
How about short-term preoperative radiotherapy $(5 \times$ 5 Gy) versus combined radiochemotherapy? Short-term radiotherapy is more convenient for the patients as the curative surgery will be just delayed by 1 week and the costs for the national health systems are reduced. Furthermore, the early toxicity rate is very low as well as the late toxicity rate, provided that a modern 4-field radiation technique is used. The Swedish Rectal Cancer Trial and the Dutch Colorectal Cancer Trial have shown a significant reduction of the local recurrence rate by short-term radiotherapy compared to surgery alone, however randomised controlled trials comparing the short-term radiotherapy with the combined preoperative radiochemotherapy are required to definitely favour one concept over the other.

The role of chemotherapy has also to be re-defined. Some trials indicate that the use of chemotherapy is responsible for increased survival due to the treatment of possible distant tumour, whereas radiotherapy controls the local disease. This has prompted some surgeons to combine preoperative short-term radiotherapy with postoperative chemotherapy in patients with positive nodal status and increased risk of distant disease. Randomised controlled trials will be necessary in the future to confirm this concept.

In conclusion, the introduction of TME has improved the quality of surgery but has also questioned many treatment concepts concerning multimodality treatment. Further large randomised trials have to be conducted to identify patients requiring additional radio- or chemotherapy, and to compare the short-term radiotherapy with the combined preoperative radiochemotherapy. 